\title{
Estratégia de educação em saúde fonoaudiológica para idosos: Relato de experiência
}

\author{
Priscilla Mayara Estrela Barbosa ${ }^{1}$, Andréa Cintia Laurindo \\ Porto $^{2}$, Moisés Andrade dos Santos de Queiroz ${ }^{3}$, Cláudia Belém \\ Moura Cabral ${ }^{3}$, Rachel Cassiano de Sousa ${ }^{3}$ e Christina César Praça \\ Brasil $^{3}$ \\ 1 Universidade Estadual do Ceará, Brasil | priscillamayara@yahoo.com.br | \\ https://orcid.org/0000-0001-9611-1343 \\ ${ }^{2}$ Hospital Geral de Fortaleza, Brasil | andrea.cintialp@gmail.com | https://orcid.org/0000- \\ 0001-9621-0480 \\ ${ }^{3}$ Universidade de Fortaleza, Brasil | fonomoises@outlook.com; \\ claudiabelemmc@gmail.com; rachelcassiano@unifor.br; cpraca@unifor.br | \\ https://orcid.org/0000-0003-4887-1377; https://orcid.org/0000-0001-7235-261X; \\ https://orcid.org/0000-0002-4849-1502; https://orcid.org/0000-0002-7741-5349
}

Resumo: Introdução: As mudanças decorrentes da velhice implicam diretamente em estruturas responsáveis pela comunicação e deglutição. Na laringe, ocorrem calcificações, impactando a fonação (presbifonia) e a deglutição (presbifagia). Na audição, há redução da acuidade (presbiacusia). A memória também apresenta alterações importantes. Objetivo: Relatar a experiência de um grupo de idosos assistidos em um Centro de Referência de Assistência Social, da região metropolitana de Fortaleza, Ceará, por meio de ações que associam saúde do idoso e Fonoaudiologia. Metodologia: As reuniões aconteceram de fevereiro a junho de 2019, com 38 idosos, de ambos os sexos, com idades de 60 a 87 anos. A fonoaudióloga do Núcleo de Assistência da Saúde da Família na Atenção Básica e acadêmicos de Fonoaudiologia prepararam ações de educação em saúde sobre os temas em questão. Os participantes eram estimulados a questionar, compartilhar experiências e esclarecer dúvidas sobre memória, voz, audição e deglutição, especialmente como o envelhecimento afeta essas funções. Diários de campo e registros escritos deram suporte à coleta de dados, que foram analisados a partir da Análise de Conteúdo na modalidade temática e interpretados à luz das teorias sobre envelhecimento saudável. Resultados: Os idosos compreendem o envelhecimento como um processo irreversível, que causa adversidades na comunicação e deglutição, levando ao distanciamento social, sentimentos de vergonha e incapacidade. Os participantes apresentaram melhoras no sentido esperado da intervenção, pois passaram a dar maior atenção às práticas educativas em saúde relativas à saúde vocal, deglutição, audição e memória. Conclusão: Conclui-se que os participantes demonstraram autonomia, ao compreender e aplicar em seu cotidiano as orientações dadas nos encontros, passando a ser multiplicadores dos novos aprendizados junto aos seus pares. Ações de educação em saúde que fortaleçam a rede de apoio aos idosos são essenciais, destacando-se a importância dos cuidados continuados e específicos voltados a esse público. Palavras-chave: Presbifagia; Presbiacusia; Memória; Presbifonia; Educação em Saúde.

\section{Speech Therapy Health Education Strategy for the Elderly: An Experience Report}

Abstract: Introduction: Changes caused by the aging imply structures responsible for communication and swallowing. In the larynx, calcifications occur, impacting phonation (presbyphonia) and swallowing (presbyphagia). In hearing, there is a reduction in acuity (presbycusis). Memory also suffers important changes. Objective: Report the experience of an elderly people group assisted in a Reference Center for Social Assistance, in the metropolitan region of Fortaleza, Ceará, through actions that associate health of the elderly and Speech and Hearing Sciences. Methodology: Meetings took place from February to June 2019 , with 38 elderly, of both sexes, aged from 60 to 87 years old. The speech therapist from the Family Health Center and students from this area prepared health education actions, which were based on the elderlys' questions on their health conditions. Participants were encouraged to discuss, share experiences and clarify doubts about memory, phonation, hearing and swallowing, especially how the aging process affects these functions. Field diaries and written records supported data collection. Data analysis based on the Content Analysis in the thematic modality and the interpretation was held in the light of theories on healthy aging Results: The elderly understand aging as an irreversible process, which causes problems in communication and swallowing, leading to social distance, feelings of shame and incapacity. The participants showed improvements from the interventions, as they began to value the educational health practices related to vocal health, swallowing, hearing and memory. Conclusion: Participants demonstrated more autonomy than before, by understanding and applying the guidelines given in the meetings in their daily lives. They became multipliers of their new learnings to their peers and families. Health education actions to strengthen the support network for the elderly are essential, highlighting the importance of continuous and specific care aimed at this population.

Keywords: Presbyphagia; Presbycusis; Memory; Presbyophonia; Health Education. 


\section{Introdução}

Nas últimas décadas, houve um aumento na expectativa de vida da população mundial, compreendendo uma transição demográfica e a inversão nas pirâmides demográficas (Martins, Nascimento, Souza, Sá, Feres, Soares, \& Ferreira, 2016). Dados do Instituto Brasileiro de Geografia e Estatística (IBGE) estimam que o número de habitantes no Brasil atingiu a marca de aproximadamente 208.5 milhões em 2018 (IBGE, 2019a). Desse contingente, $15,4 \%$ estão na faixa etária de 60 anos ou mais (IBGE, 2019b). O aumento superou as estimativas previstas para o número de idosos no Brasil em 2020 (Simões, 2016), constando-se que a população com 60 anos ou mais compreende mais de 32 milhões de habitantes, reiterando o acelerado envelhecimento populacional no país.

O envelhecimento é um processo dinâmico, progressivo e irreversível, variando de acordo com: estilo de vida, condições socioeconômicas e de saúde (Pinto, Reis, Almeida-Brasil, Silveira, Lima, \& Ceccato, 2016). Como consequência, prejuízos nos sistemas sensoriais podem ocorrer (Lord, Delbaere, \& Sturnieks, 2018). As mudanças orgânicas decorrentes da velhice podem implicar diretamente na função laríngea, a partir de calcificações nas cartilagens que alteram a fonação. Isto imprime características próprias na voz do idoso, denominando-se presbifonia. Alterações na deglutição (presbifagia) também são decorrentes das alterações laríngeas nessa fase (Kost \& Sataloff, 2018).

Outra alteração decorrente do evelhecimento é a presbiacusia (perda progressiva da acuidade auditiva). Com o avanço da idade, o cérebro também evidencia prejuízos decorrentes da significativa perda de neurônios, a exemplo da perda de memória, levando às demências (Chern \& Golub, 2019).

Nesse contexto, o fonoaudiólogo é o profissional capacitado para acompanhar e postergar o avanço das condições clínicas que impactam a comunicação e a deglutição, além de cuidar de outros fatores relevantes para a saúde do idoso. Isto auxilia na promoção da saúde dessa população, confirmando sua importância na Atenção Primária à Saúde - APS (Guckert, Souza, \& Arakawa-Belaunde, 2020).

A dificuldade em aceitar o envelhecimento pode provocar no indivíduo negação, vergonha e distanciamento da sociedade. Com isso, há a necessidade de ações que auxiliem na melhoria da qualidade de vida do idoso, a partir de atividades laborais e recreativas, individuais ou grupais, capazes de proporcionar maior interação social e interpessoal, além de apoiar a aceitação das limitações da senescência (Silva, Assunção, \& Porto, 2020). Essas estratégias objetivam promover saúde, prevenir agravos, retardar o aparecimento de doenças, reduzir as fragilidades, bem como estimular e manter a independência e a autonomia dos idosos (Santos, Feitosa, Melo, \& Canuto, 2018). Salienta-se a importância da abordagem integral e interdisciplinar pelos profissionais da saúde, considerando os fatores físicos, psicológicos, ambientais e socioculturais que influenciam a saúde do idoso (Fonseca, Estevam, Mariz, Oliveira, \& Souza, 2021).

Os grupos de idosos com foco na Promoção da Saúde (PS) e na prevenção de agravos constituem uma estratégia da Atenção Básica $(A B)$ à Saúde e da Assistência Social. Nesse sentido, o Centro de Referência de Assistência Social (CRAS) é a porta de entrada para a resolubilidade de muitos problemas socais, por ser um serviço público para o fortalecimento das relações entre indivíduo, família e comunidade. Promove também a organização e a articulação das unidades da rede socioassistencial e de outras políticas (Carneiro, Gomes, Durães, Jesus, Chaves, Lima, Costa, \& Caldeira, 2020).

Assim, o CRAS, ao promover a intersetorialidade e albergar parcerias entre os setores que assistem a população, é o ambiente ideal para a realização dos Grupos de Promoção de Saúde (GPS), que constituem intervenções coletivas e interdisciplinares para o estímulo a interação e a cooperação.

Nessa perspectiva, constituiu-se em 2014 e perdura até os dias atuais (2021), um grupo de idosos organizado pelas psicólogas e assistentes sociais de um CRAS, em Caucaia, Ceará, Brasil. Para isso, estabeleceu-se uma parceria entre a Estratégia Saúde da Família (ESF) e o Núcleo Ampliado de Saúde da Família e Atenção Básica (NASF-AB). 
Os encontros grupais com os idosos são conduzidos e supervisionados pelos profissionais que atuam em uma Unidade de Atenção Primária à (UAPS) que assiste a população que frequenta o referido CRAS.

A UAPS em pauta conta com quatro equipes da Estratégia Saúde da Família (ESF) e uma equipe do NASF-AB, sendo composta por terapeutas ocupacionais, fonoaudióloga, fisioterapeuta, psicóloga, farmacêutica, nutricionistas, educadora física e assistente social.

Os encontros com os idosos ocorrem na segunda sexta-feira de cada mês, pela manhã, no CRAS sob investigação. Cada encontro é organizado por um profissional da saúde com a cooperação dos funcionários do serviço. Os temas abordados emergem das inquietações e dúvidas dos participantes, contemplando assuntos selecionados mediante votação realizada ao final de cada reunião. Ao final de cada encontro, há uma roda de conversa, na qual os participantes discutem, trocam experiências e conhecimentos.

Diante do exposto, esta investigação respalda-se no seguinte questionamento: como uma estratégia de educação em saúde voltada a um grupo de idosos assistidos em um CRAS pode contribuir com a saúde fonoaudiológica dessa população?

O artigo objetiva relatar a experiência de um grupo de idosos assistidos em um CRAS por meio de ações de educação em saúde que associam saúde do idoso e Fonoaudiologia.

\section{Metodologia}

Realizou-se um estudo descritivo, qualitativo, do tipo relato de experiência. Segundo Minayo, (2014), a pesquisa qualitativa permite a reflexão sobre fatos, significados, motivos, aspirações, crenças, valores e atitudes que compreendem as relações, os processos e os fenômenos. Ressalta-se que não estabelece previamente a amostra e que os pressupostos são afirmações provisórias sobre um problema.

Os encontros do grupo de idosos foram realizados de fevereiro a junho de 2019, no turno manhã, no CRAS sob investigação. Participaram pessoas de ambos os sexos, com idades de 60 a 89 anos. Todos os idosos moradores da área adstrita podiam participar dos encontros, mediante a comprovação de endereço, como requisito para a vinculação às atividades. Durante o período de coleta de dados, frequentavam as reuniões 38 idosos.

Nas exposições dos temas para o grupo de idosos, havia, pelo menos, um assistente social que atua no CRAS, responsável por dirigir a acolhida, a organização geral do evento e a conclusão da reunião.

Uma intervenção fonoaudióloga foi requisitada pelos organizadores para abordar temas de interesse dos idosos, que se basearam em relatos e dúvidas sobre: memória, voz, audição e deglutição. Requereu-se apresentar como o envelhecimento afeta essas funções e medidas promotoras da saúde. Estabeleceram-se quatro encontros para abordar as temáticas relativas a saúde do idoso e Fonoaudiologia.

Os investigadores foram uma fonoaudióloga do NASF - AB e três acadêmicos de Fonoaudiologia de uma instituição de ensino superior. Estes prepararam palestras e ações, seguindo as sugestões dos participantes e dos profissionais do CRAS e do NASF$A B$. Os investigadores também conduziram as quatro oficinas como facilitadores e observadores. Houve uma reunião de planejamento entre as equipes para definir a programação: acolhimento, palestra expositiva e interativa, lanche saudável e socialização.

Todo a explanação foi pensada de maneira a facilitar a compreensão dos idosos, com linguagem acessível. Cada encontro durou, em mádia, três horas e meia. Os idosos que apresentaram queixas relacionadas a saúde comunicativa e/ou deglutição foram acolhidos e agendados para posterior consulta com a fonoaudióloga do NASF-AB. 
Salienta-se que as exposições se desenvolveram com metodologias participativas, na quais procurou-se preservar a identidade social e cultural dos integrantes, valorizando saberes prévios e unindo o conhecimento popular aos conteúdos de saúde vocal, memória, deglutição e audição. Todos os instrumentos utilizados foram elaborados pelos acadêmicos de Fonoaudiologia com orientações da fonoaudióloga do NASF-AB.

Para promover hábitos mastigatórios saudáveis, melhoras na audição, treinamentos vocais e de memória, os encontros foram realizados como oficinas. Estas iniciaram com exposições dialogadas sobre outros assuntos em pauta. Em seguida, os participantes expunham suas dúvidas e as discutiam com os facilitadores. O círculo de cultura de Paulo Freire também foi utilizado nestas ocasiões (Freire, 2013).

Tal método foi oportuno para este estudo, pois favorece uma relação dialógica do expositor com os ouvintes, por ser capaz de demonstrar o que estava escondido e permitir que as reflexões, dúvidas e queixas dos participantes levem todos juntos a novas propostas de intervenção. Essa estratégia alinha-se ao escopo das pesquisas qualitativas, pois são uma maneira de compreender, fixar e propagar o saber (Freire, 2013).

Realizou-se observação participante das oficinas, utilizando-se diários de campo e registos escritos para a coleta de dados. Em cada encontro, os acadêmicos de Fonoaudiologia (facilitadores e observadores) anotaram as principais dúvidas e experiências compartilhadas. Reações emocionais, expressões ou movimentos (imitação ou reação) dos idosos também foram registados por escrito para análise.

Após os encontros, a equipe de Fonoaudiologia reunia-se a analisava os dados e categorizava em temáticas, a partir da Análise de Conteúdo na modalidade temática (Minayo, Deslandes, \& Gomes, 2013). Esse método propiciou a leitura em profundidade, a apropriação do material, a revisita à experiência vivenciada e a categorização das principais dúvidas dos idosos com relação a saúde fonoaudiológica, as quais convergem com os assuntos abordados nos encontros, desdobrando-se nas seguintes temáticas: voz, deglutição, audição e memória. A interpretação dos achados foi embasada na literatura que versa sobre o envelhecimento saudável.

Como se trata de um relato de experiência, considerando as perspectivas dos investigadores, este estudo não requer aprovação pelo Comitê de Ética.

\section{Resultados e Discussão}

Ao explorar o campo, inicialmente, os pesquisadores observaram que as estratégias e ações de prevenção e promoção de saúde realizadas pelas equipes de saúde e do CRAS junto ao idoso devem ser ampliadas e incentivadas, uma vez que sses procedimentos facilitam a vida dos idosos, ampliando o bem-estar e a qualidade de vida (física, psicológica, ambiental e social). Ademais, isto pode melhorar a extensão do suporte social percebido. A Política Nacional de Atenção à Saúde, instituída em 28 de setembro de 2004, teve o objetivo de desenvolver ações que possibilitem a promoção da qualidade de vida, a educação em saúde, além da proteção e recuperação da saúde de indivíduos e comunidades (Brasil, 2013).

As ações de educação em saúde voltadas ao idoso, buscando esclarecer sobre a saúde da comunicação e da deglutição auxiliam no processo de corresponsabilização pela saúde e na manutenção de hábitos saudáveis. Ademais, conhecer os problemas que o envelhecimento traz para a voz, deglutição, audição e memória, facilita a identificação prococe e a adoção de medidas de controle e prevenção. 
É muito comum os idosos apresentarem dúvidas sobre as mudanças que acometem a voz com o avanço da idade, chegando a caracterizar a voz da seguinte forma: " $A$ voz vai enfraquecendo [com o avanço da idade] ...", "Já não canto como antes", "Ao falar muito, fico cansado...", "A voz da mulher idosa parece mais grossa e a do homem idoso fica mais fina, não é?"

Quanto a presbifonia, explicou-se aos idosos que se trata de uma alteração vocal que ocorre em idosos, causada pelo envelhecimento das estruturas laríngeas. O início da decadência vocal pode ser decorrente de diversos fatores relacionados tanto à saúde física quanto à mental (Brown, Zhou, \& Husain, 2020). Muitos idosos revelaram perceber, com o passar dos anos, que a voz fica mais fraca para ambos os sexos; para mulheres, torna-se mais grave, e, para homens, mais aguda. Entretanto, não foi um aspecto percebido amplamente pelos participantes. O que realmente declaram é que " $A$ voz muda com o tempo", "Não tenho mais a voz que eu tinha aos 20 ou 40 anos. Agora é diferente".

Os facilitadores dos encontros apresentaram noções básicas sobre a produção vocal, fatores de risco, sintomas vocais mais frequentes, exercícios de aquecimento e desaquecimento vocal, além de medidas simples de hidratação e higiene vocal.

A presbiacusia revelou-se como uma das mais frequentes preocupações dos idosos, pois é uma alteração facilmente perceptível e que impacta a qualidade da comunicação. Alguns idosos expressaram que "Ficar sem escutar é uma tormenta..."

Não "escutar direito" pode levar o indivíduo a afastar-se de suas funções sociais e ao isolamento. A presbiacusia tem um padrão audiológico com características de uma perda neurossensorial bilateral e gradualmente progressiva, o que é identificado pelo próprio indivíduo. Isto influencia a sua qualidade de vida, devido aos déficits na percepção da fala e prejuízos na cognição, levando-o a restrição na participação social, progressão do isolamento e falta de socialização (Jafari, Kolb, \& Mohajerani, 2019).

Nos encontros, apresentaram-se estratégias de identificação da presbiacusia, incluindo as alternativas de correção e acompanhamento da evolução. Próteses auditivas, leitura labial e foco no interlocutor foram algumas medidas exploradas e esclarecidas aos presentes.

A perda da memória é outro aspecto que chama a atenção do idoso e traz preocupação. $\mathrm{O}$ fato de não lembrar nomes de pessoas conhecidas, não lembrar se tomou ou não uma medicação, ou mesmo se já executou alguma tarefa cotidiana causa desconforto e insegurança, segundo os participantes deste estudo. Destaca-se que, durante o processo de envelhecimento, são comuns os episódios de esquecimento, mas, de acordo com a frequência, estes podem prejudicar a autoestima, o convívio social e a execução de tarefas. Explanou-se aos idosos que isto decorre da própria fisiologia humana, a partir da diminuição da funcionalidade cerebral. Esse déficit progressivo da memória e de outras habilidades amplia a predisposição às alterações cognitivas, além de acarretar a incidência de demência ou de outras intercorrências (Loughrey, Kelly, Kelley, Brennan, \& Lawlor BA, 2018).

Os fonoaudiólogos apresentaram estratégias de estimulação da memória, a partir de leituras, jogos, palavras-cruzadas, dentre outros recursos. Orientaram, ainda, sobre a ida ao médico para o acompanhamento periódico e a tomada de medidas terapêuticas em caso de necessidade.

No tocante a presbifagia, muitos idosos relatam episódios de engasgo com a própria saliva ou algumas consistências alimentares. Muitas vezes, esses episódios são subestimados pelos idosos ou familiares ("É só um engasgo simples... tossiu, passa..."), porém o seu agravamento pode levar a repetidas intercorrências de saúde, o que inclui as peneumonias repetitivas por aspiração de alimentos. As intercorrências na deglutição podem afastar o indivíduo da sociedade, pois os afetados sentem receio de comer em público, uma vez que esses sintomas os caracterizam como doentes e incapacitados para si e para a família, requerendo cuidados psicológicos e físicos (Warnecke, Dziewas, Wirth, Bauer, \& Prell, 2019). 
$\mathrm{Na}$ oficina sobre presbifagia, foram apresentadas estratégias facilitadoras para evitar engasgos e oferecer maior conforto e segurança durante a alimentação. Assim, demonstraram-se posturas alimentares, volume de ingesta de alimentos e consistências para dar ao idoso maior autonomia e proteção durante a deglutição.

Percebeu-se, durante os encontros, que os participantes possuíam conhecimento muito limitado sobre os quatro temas abordados, o que ocasionava angústia e medo dos agravos. Ademais, as orientações oferecidas foram bem recebidas pelos idosos e remeteram a sensação de segurança e autocontrole de sua saúde.

Os profissionais do CRAS, assim como os idosos que compareceram aos encontros, relataram que, após as intervenções, o público-alvo apresentou aumento nas práticas de autocuidado, dando continuidade à proposta de prevenção de doenças e promoção de saúde. Houve também um monitoramento das práticas abordadas nos encontros, o que foi feito pelos Agentes Comunitários de Saúde (ACS) da área assistida e por um idoso voluntário. Estes deram continuidade às orientações nos domicílios, o que se caracterizou como reforço às orientações sobre cuidados contínuos.

No decorrer das ações sociais e educativas, após a coleta de dados, os participantes relataram ter adquirido e empregado os conhecimentos obtidos sobre os cuidados com a saúde vocal, deglutição, audição e memória. Aqueles que apresentaram queixas sobre os assuntos abordados, foram encaminhados pelos facilitadores a fonoaudiólogos para avaliação e cuidados específicos.

Com esta vivência, notou-se que há a necessidade de dar prosseguimento às intervenções sobre saúde fonoaudiológica no âmbito do envelhecimento, além de abordar outras temáticas transdisciplinares, pois as estratégias adotadas estimulam o autocuidado. Os processos de educação em saúde buscam ampliar e fortalecer a autonomia social, muitas vezes, prejudicada na terceira idade (Barbosa, Martin, \& Oliveira, 2020).

No decorrer dos encontros, observaram-se idosos mais orientados quanto aos temas abordados, saudáveis e autônomos em relação à sua saúde, além de conscientes sobre os aspectos da velhice e de como evitar, aceitar e tratar alterações relacionadas à memória, voz, deglutição e audição.

O envelhecimento ainda é visto por muitas pessoas como um estado patológico. A partir dos encontros, percebeu-se a importância de ter profissionais de saúde capacitados para lidar com a população idosa e suas necessidades, incluindo o fonoaudiólogo. Nesse sentido, a educação em saúde é o processo por meio do qual os profissionais de saúde participam do cotidiano da população, constituindo uma uma prática social crítica e transformadora, perceptivelmente indispensável (Farias, Assis, Dantas, Silva, Monteiro, Carvalho, Fernandes, \& Bezerra, 2020).

\section{Conclusões}

Os resultados da intervenção mostraram que os objetivos dos encontros foram alcançados. A análise dos dados permitiu concluir que os participantes apresentaram melhoras no sentido esperado da intervenção, pois passaram a dar maior atenção às práticas educativas em saúde relativas à saúde vocal, deglutição, audição e memória. Verificou-se, ainda, que os idosos demonstraram maior autonomia, ao compreender e aplicar em seu cotidiano as orientações fonoaudiológicas.

Cabe ressaltar que são escassos os relatos sobre abordagens de promoção da saúde em parceria com os CRAS, por isso, sugere-se que haja mais intervenções dessa natureza para ampliar a disseminação de conhecimento em saúde para a população, como forma de prevenir doenças e aumentar a qualidade de vida dos idosos.

Ações futuras podem utilizar medidas observacionais para averiguar e melhor acompanhar as vivências dos conhecimentos obtidos, registar mudanças ao longo da intervenção ou indicadores de satisfação do paciente. 
Análises desse tipo auxiliam na interpretação dos resultados para melhor ajustar a metodologia dos encontros ao público-alvo, bem como reduzir as resistências na adesão aos hábitos saudáveis. Assim, tal estudo pode trazer importantes contribuições para o planejamento de ações futuras, adequando-as ao contexto e à população-alvo.

Os ganhos advindos de novos estudos que associem fonoaudiologia e saúde do idoso poderão fortalecer estratégias promotoras de saúde, interessando aos profissionais da saúde e da assistência social, pesquisadores, gestores, além de contribuir com as políticas públicas de educação, saúde e assistência social voltadas ao idoso.

A adoção de uma abordagem de investigação qualitativa permitiu verificar que os encontros com os idosos foram percebidos pelos participantes como proveitosos para a melhoria das condições de saúde do grupo, uma vez que propiciam o autocuidado e a autonomia. Essas evidências foram destacadas a partir dos registos nos diários de campo e discussões entre os facilitadores, o que favoreceu a análise de conteúdo e a interpretação das temáticas. Nessa perspectiva, a implantação de ações dessa natureza em outras UAPS e CRAS poderá atingir um número significativo da população e capacitála para atuar como uma rede de apoio social promotora de saúde.

Pode-se concluir que o envelhecimento saudável é resultado da interação multidimensional entre saúde física, saúde mental, integração social, suporte familiar e independência econômica. Para isso, fazem-se necessárias ações contínuas de educação em saúde que fortaleçam a rede de apoio aos idosos, além de destacar a importância de cuidados continuados e específicos voltados a esse público.

\section{Referências}

Barbosa, W. G., Martin, D. S., \& Oliveira, A. F. (2020). Cultura da educação em saúde na prevenção de doenças em idosos. Revista OKARA: Geografia em debate, 14(1), 213-225.

Brasil. (2003). Lei no 10741 de 3 de outubro de 2003. Dispõe sobre o Estatuto do Idoso. http://www.planalto.gov.br/ccivil_03/leis/2003/110.741.htm

Brown, H. J., Zhou, D., \& Husain, I. A. (2020). Management of presbyphonia: A systematic review of the efficacy of surgical intervention. Am J Otolaryngol., 41(4), 102532.

Carneiro, J. A., Gomes, C. A. D., Durães, W., Jesus, D. R., Chaves, K. L. L., Lima, C. A., Costa, F. M., \& Caldeira, A. P. (2020). Autopercepção negativa da saúde: prevalência e fatores associados entre idosos assistidos em centro de referência. Ciência \& Saúde Coletiva, 25(3), 909-918.

Chern, A., \& Golub, J. S. (2019). Age-related Hearing Loss and Dementia. Alzheimer Dis Assoc Disord., 33(3), 285-290.

Farias, L. L. S., Assis, L. T. D., Dantas, R. B., Silva, F. C. S., Monteiro, V. C. M., Carvalho, P. R. S., Fernandes, C. C., \& Bezerra, I. N. M. (2020). Ações de promoção à saúde vivenciada com grupo de isoso: um relato de experiência. Brazilian Journal of Development, 6(6), 3381733828.

Fonseca, A. C. D., Estevam, S. R., Mariz, S. L. L., Oliveira, L. C., \& Souza, C. M. P. (2021). Interdisciplinaridade na gestão do cuidado ao idoso. Brazilian Journal of Health Review, 4(2), 4045-4050.

Freire, P. (2013). Educação como prática da liberdade. 44. ed., Rio de Janeiro: Paz e Terra.

Guckert, S. B., Souza, C. R., \& Arakawa-Belaunde, A. M. (2020). Atuação fonoaudiológica na atenção básica na perspectiva de profissionais dos núcleos de apoio à saúde da família. CoDAS, 32(5), e20190102.

Instituto Brasileiro de Geografia e Estatística (IBGE). (2019a). SIDRA: Estimativas de População EstimaPop. Rio de Janeiro: IBGE. https://sidra.ibge.gov.br/pesquisa/EstimaPop/tabelas

Instituto Brasileiro de Geografia e Estatística (IBGE), Diretoria de Pesquisas, Coordenação de Trabalho e Rendimento. (2019b). Pesquisa Nacional por Amostra de Domicílios Contínua Características gerais dos domicílios e dos moradores 2018. Rio de Janeiro: IBGE. https://biblioteca.ibge.gov.br/visualizacao/livros/liv101654_informativo.pdf 
Jafari, Z., Kolb, B. E., \& Mohajerani, M. H. (2019). Age-related hearing loss and tinnitus, dementia risk, and auditory amplification outcomes. Ageing Res Rev., 56, 100963.

Lord, S. R., Delbaere, K., \& Sturnieks, D. L. (2018). Aging. Handb Clin Neurol., 159, 157-171.

Loughrey, D. G., Kelly, M. E., Kelley, G. A., Brennan, S., \& Lawlor, B. A. (2018). Association of Age-Related Hearing Loss With Cognitive Function, Cognitive Impairment, and Dementia: A Systematic Review and Meta-analysis. JAMA Otolaryngol Head Neck Surg., 144(2), 115-126.

Kost, K. M., \& Sataloff, R. T. (2018). Voice Disorders in the Elderly. Clin Geriatr Med., 34(2), 191203.

Martins, A. M. E. D. B. L., Nascimento, J. E., Souza, J. G. S., Sá, M. A. B. D., Feres, S. D. B. L., Soares, B. P., \& Ferreira, E. F. (2016). Associação entre transtornos mentais comuns e condições subjetivas de saúde entre idosos. Ciência \& Saúde Coletiva, 21(11), 3387-3398.

Minayo, M. C. S. (2014). O desafio do conhecimento: pesquisa qualitativa em saúde. 14. ed., São Paulo: Hucitec.

Minayo, M. C. S., Deslandes, S. F., \& Gomes, R. (2013). Pesquisa social: teoria, método e criatividade. 33. ed. Petrópolis: Vozes.

Pinto, I. V. L., Reis, A. M. M., Almeida-Brasil, C. C., Silveira, M. R., Lima, M. G., \& Ceccato, M. G. B. (2016). Avaliação da compreensão da farmacoterapia entre idosos atendidos na Atenção Primária à Saúde de Belo Horizonte, MG, Brasil. Ciência \& Saúde Coletiva, 21(11), 34693481.

Santos, R. G. O., Feitosa, A. L. F., Melo, A. M. S., \& Canuto, M. S. B. (2018). Fonoaudiologia e Gerontologia: revisão sistemática da atuação Fonoaudiológica. Distúrb Comun., 30(4), 748 758.

Silva, S. G. M., Assunção, A. N., \& Porto, V. F. A. (2020). Perfil fonoaudiológico de idosos participantes de grupos de convivência desenvolvidos na Atenção Primária à Saúde. Distúrb Comun., 32(2), 245-258.

Simões, C. C. S. (2016). Relações entre as alterações históricas na dinâmica demográfica brasileira e os impactos decorrentes do processo de envelhecimento da população. Rio de Janeiro: IBGE, Coordenação de População e Indicadores Sociais.

Warnecke, T., Dziewas, R., Wirth, R., Bauer, J. M., \& Prell, T. Z. (2019). Dysphagia from a neurogeriatric point of view : Pathogenesis, diagnosis and management. Gerontol Geriatr., 52(4), 330-335 